
allemande

\title{
1917 - Narrationen eines Wendepunkts in österreichischen Geschichtslehrwerken und in Ausstellungen
}

Andrea Brait

\section{(2) OpenEdition}

Journals

Édition électronique

URL : https://journals.openedition.org/allemagne/600

DOI : $10.4000 /$ allemagne. 600

ISSN : 2605-7913

Éditeur

Société d'études allemandes

Édition imprimée

Date de publication : 29 décembre 2017

Pagination : 447-462

ISSN : 0035-0974

Référence électronique

Andrea Brait, „1917 - Narrationen eines Wendepunkts in österreichischen Geschichtslehrwerken und in Ausstellungen ", Revue d'Allemagne et des pays de langue allemande [Online], 49-2 | 2017, Online erschienen am: 29 Dezember 2018, abgerufen am 19 Mai 2021. URL: http://journals.openedition.org/ allemagne/600 ; DOl: https://doi.org/10.4000/allemagne.600 


\title{
1917 - Narrationen eines Wendepunkts in österreichischen Geschichtslehrwerken und in Ausstellungen
}

\author{
- Andrea Brait*
}

Eine Umfrage der Redaktionen von ZIB2 und ORF.at unter österreichischen Historikerinnen und Historikern brachte das klare Ergebnis, dass für das 20. Jahrhundert drei Schlüsseljahre zu identifizieren seien: 1917, 1945 und 1989. Der Grazer Historiker Helmut Konrad meinte im Zuge der Umfrage etwa zu 1917: „Das Schlüsseljahr im Ersten Weltkrieg. Beginn der bipolaren Welt“(1). Die folgende Untersuchung beschäftigt sich damit, wie das Jahr 1917 in österreichischen Schulbüchern und Ausstellungen bewertet wird ${ }^{(2)}$. Findet sich auch dort eine Betitelung als Schlüsseljahr oder Epochenjahr und wie werden die Einschätzungen erklärt?

Ausstellungen zeigen ähnlich wie Geschichtslehrwerke ein an ein Laienpublikum gerichtetes Narrativ. Da sie aus dem breiten Forschungsstand aufgrund des begrenzten Platzes notwendigerweise eine Auswahl treffen müssen, zeigen sie, so die Grundannahme der Untersuchung, welche historischen Entwicklungen von einer Gesellschaft zu einer bestimmten Zeit einen Platz im Funktionsgedächtnis ${ }^{(3)}$ haben.

\section{Österreich-Ungarn im Jahr 1917}

Bei der Suche nach einem „Beginn“ des Zeitraumes, der durch die Zeitgeschichte zu erforschen sei, wurde in den 1950er-Jahren das Jahr 1917 gefunden. Zeitgeschichte sei, so Martin Broszat im Jahr 1957, die „Geschichte der mit dem Jahr 1917

* Assistenzprofessorin am Institut für Zeitgeschichte und am Institut für Fachdidaktik, Universität Innsbruck.

1 http://orf.at/stories/2211176/2211206/ (online am 2. Jänner 2014).

2 Um die Vergleichbarkeit der verschiedenen Geschichtsdarstellungen zu gewährleisten, konzentriert sich die Analyse auf die Interpretation in Form von Texten.

3 Aleida Assmann, „Funktions- und Speichergedächtnis. Zwei Modi der Erinnerung“, in: Kristin Platt, Mihran Dabag (Hg.), Generation und Gedächtnis. Erinnerungen und kollektive Identitäten, Opladen, 1995, S. 169-185. 
sich abzeichnenden neuen universalgeschichtlichen Periode“(4). Auch nach diversen Diskussionen um eine Definition der Zeitgeschichte bleibt das Jahr 1917 als Zäsur weiterhin relevant. So wurde etwa vorgeschlagen, die Zeitgeschichte in eine ältere Phase von 1917 bis 1945, eine jüngere von 1945 bis 1989 und eine jüngste von 1990 bis heute zu unterteilen ${ }^{(5)}$, womit die von Hans Rothfels, der als Begründer der deutschen Zeitgeschichte gilt, vorgeschlagene Definition als „Epoche der Mitlebenden“(6) zunehmend in Frage gestellt wird ${ }^{(7)}$. Begründet wird die Zäsur von der internationalen Historikerzunft immer wieder mit der Änderung der Machtverhältnisse im Russischen Reich, wobei in vielen Analysen zum Ersten Weltkrieg gleichsam das darauffolgende Ausscheiden aus diesem Krieg sowie der Kriegseintritt der USA als zentrale Ereignisse genannt werden, welche den Kriegsausgang beeinflussten und die Weltgeschichte zweifelsohne nachhaltig veränderten ${ }^{(8)}$.

Für die Entwicklung Österreich(-Ungarns) sind hinsichtlich der Bedeutung des Jahres 1917 noch einige weitere, (den Weltkrieg mit-)entscheidende Entwicklungen zu nennen, allen voran die unter dem Schlagwort „Sixtus-Briefe“ bekannt gewordenen Friedensbemühungen von Kaiser Karl, die Wiedereröffnung des Reichsrates und die Zwölfte Isonzoschlacht.

Im Verlauf des Jahres 1917 unternahm der österreichische Kaiser Karl I. mehrere Versuche, den Krieg zu beenden. In der Sitzung des österreichisch-ungarischen Ministerrates vom 12. Jänner 1917 verlangte er, dass Friedensziele ausgearbeitet werden ${ }^{(9)}$. Über seinen Schwager Sixtus von Bourbon-Parma versuchte er schließlich, Verbindung zu Frankreich aufzunehmen, um die Bedingungen für einen Frieden zu erkunden. Im ersten Brief vom 24. März 1917, dessen Original verschwunden ist, sprach er sich für die Souveränität von Belgien und Serbien aus und versprach die französischen Forderungen nach Rückgabe Elsaß-Lothringens, das zum Deutschen Reich gehörte, zu unterstützen. Im zweiten Brief vom 9. Mai 1917 ging es vor allem um die italienischen Gebietsansprüche, die Karl später überprüfen wollte. Die beiden Briefe führten jedoch aufgrund unterschiedlicher Interessen der Entente-Mächte zu keinen Friedensgesprächen. Im Frühjahr 1918 wurden die Friedensbemühungen öffentlich und Karl sah sich gezwungen, sein Vorgehen zu leugnen. Am Ende der Affäre, im Zuge derer der k. k. Minister des kaiserlichen Hauses und des Äußeren Ottokar Graf Czernin demissionierte, war die Glaubwürdigkeit des Kaisers, vor allem

4 Martin Broszat, „Aufgaben und Probleme zeitgeschichtlichen Unterrichts“ (1957), in: Hermann Graml, Klaus-Dietmar Henke (Hg.), Nach Hitler. Der schwierige Umgang mit unserer Geschichte. Beiträge von Martin Broszat, München, 1987, S. 9-35, hier S. 10.

5 Martin SABrow, Der Zeitraum der Zeitgeschichte, http://www.zzf-pdm.de/Portals/_Rainbow/images/ mitarbeiter/Vortrag_Martin_Sabrow_Der_Zeitraum_der_Zeitgeschichte_01_10_2014_Paris.pdf (online am 14. Mai 2016), S. 3.

6 Hans Rothғels, „Zeitgeschichte als Aufgabe“, Vierteljahreshefte für Zeitgeschichte, 1/1 (1953), S. 1-8, hier S. 2.

7 Andreas Wirsching, „,Epoche der Mitlebenden“ - Kritik einer Epoche“, Zeithistorische Forschungen/ Studies in Contemporary History, 8 (2011), S. 150-155 hier S. 151.

8 U.a. Lutz Raphael, „Imperiale Gewalt und mobilisierte Nation. Europa 1914-1945“, München, 2011, S. 43 .

9 Manfried Rauchensteiner, Josef Broukal, Der Erste Weltkrieg und das Ende der Habsburgermonarchie in aller Kürze, Wien/Köln/Weimar, 2015, S. 142. 
in Deutschland, erschüttert. Karl musste einer noch engeren militärischen Bindung seines Reiches an den deutschen Bündnispartner zustimmen ${ }^{(10)}$.

Als der Krieg im Sommer 1914 begann, war der Reichsrat gerade vertagt - und er sollte nach der Sommerpause auch nicht wieder einberufen werden. Regiert wurde mit Hilfe des $\$ 14$ des Staatsgrundgesetzes vom 21. Dezember 1867, der dem Kaiser ein alleiniges Gesetzgebungsrecht zusprach, wenn der Reichsrat nicht versammelt ist ${ }^{(11)}$. Erst am 30. Mai 1917 sollte der Reichsrat zu seiner nächsten Sitzung zusammentreten. Berthold Sutter und Ernst Bruckmüller zufolge wollte Karl „mit der Wiedereinberufung des Parlaments eine Geste des guten Willens setzen“(12). Der Einsatz der Notverordnungen wurde im Reichsrat massiv kritisiert ${ }^{(13)}$. Dennoch wurde im gleichen Jahr das Kriegswirtschaftliche Ermächtigungsgesetz ${ }^{(14)}$ beschlossen, das im Jahr 1933 unter Bundeskanzler Engelbert Dollfuß benutzt wurde, um ohne Parlament zu regieren ${ }^{(15)}$. Der Reichsrat wurde in den letzten Monaten des Krieges insbesondere zum Schauplatz der Nationalitätenkonflikte der Monarchie. Manfried Rauchensteiner zufolge wurde bereits in der Eröffnungssitzung klar, dass sich die Nationalitäten vom Gesamtstaat lossagen wollten ${ }^{(16)}$.

1917 war außerdem jenes Jahr, in dem der österreichisch-ungarischen Armee in der 12. Isonzoschlacht mit Unterstützung deutscher Truppen ein Erfolg an der Südwestfront gelang, nachdem die italienischen Truppen in den elf vorherigen Schlachten immer mehr Gebiete erobert hatten. Der Angriff begann am Morgen des 24. Oktober am oberen Isonzo zwischen Flitsch (Bovec) und Tolmein (Tolmin) mit einem enormen Artilleriefeuer und mit Giftgas. Schnell konnten nennenswerte Erfolge und Gebietsgewinne erzielt werden. Das eigentliche Maximalziel, der Tagliamento, wurde

10 U.a. Tamara Griesser-Pecar, Die Mission Sixtus. Österreichs Friedensversuch im Ersten Weltkrieg, Wien/München, 1988; Manfried Rauchensteiner, „,Ich habe erfahren, daß mein Kaiser lügt.' Die ,Sixtus-Affäre‘ 1917/18“, in: Michael Gehler, Hubert Sickinger (Hg.), Politische Affären und Skandale in Österreich. Von Mayerling bis Waldheim, Innsbruck/Wien/Bozen, 2007, S. 148-169.

$11 \$ 14$ Gesetz, wodurch das Grundgesetz über die Reichsvertretung vom 26. Februar 1861 abgeändert wird (RGBl. 11/1867).

12 Berthold Sutter, Ernst Bruckmüller, „Der Reichsrat, das Parlament der westlichen Reichshälfte Österreich-Ungarns“, in: Ernst Bruckmüller (Hg.), Parlamentarismus in Österreich (Schriften des Instituts für Österreichkunde, 64), Wien, 2001, S. 60-109, hier S. 95.

13 Der Sozialdemokrat Karl Seitz betonte: „Nicht eine Zeile dieser $\$ 14$-Verordnungen ist wert zu einem dauernden Besitzstande zu werden“, wofür er dem Protokoll zufolge „lebenhafte[n] Beifall und Händeklatschen“ erntete. Stenografische Protokolle des Reichsrates, Haus der Abgeordneten, 5. Sitzung der XXII. Session am 13. Juni 1917, S. 183.

14 Gesetz vom 24. Juli 1917, mit dem die Regierung ermächtigt wird, aus Anlaß der durch den Kriegszustand verursachten außerordentlichen Verhältnisse die notwendigen Verfügungen auf wirtschaftlichem Gebiete zu treffen (RGB1. 307/1917). Dieses basierte auf: Kaiserliche Verordnung, mit welcher die Regierung ermächtigt wird, aus Anlaß der durch den Kriegszustand verursachten außerordentlichen Verhältnisse die notwendigen Verfügungen auf wirtschaftlichem Gebiete zu treffen (RGBl. 274/1914).

15 Hannes Leidinger, Verena Moritz, „Das Kriegswirtschaftliche Ermächtigungsgesetz (KWEG) vor dem Hintergrund der österreichischen Verfassungsentwicklung“, in: Florian Wenninger, Lucile Dreidemy (Hg.), Das Dollfuß/Schuschnigg-Regime 1933-1938. Vermessung eines Forschungsfeldes, Wien/Köln/Weimar, 2013, S. 449-470.

16 Manfried Rauchensteiner, Der Erste Weltkrieg und das Ende der Habsburgermonarchie, Wien/ Köln/Weimar, 2013, S. 737. 
überschritten, der Vormarsch kam erst am Piave zum Stehen, wo eine neue, viel kürzere Frontlinie entstand. Die erbeuteten Vorräte sowie zahlreiche Plünderungen in den eroberten Gebieten halfen den Truppen im folgenden Winter - es wurden sogar große Mengen nach Österreich und Deutschland transportiert, die dort dringend benötigt wurden, da die Offensive einen großen Teil der verfügbaren Ressourcen verschlungen hatte. Das große Ziel, die Einkesselung der italienischen Truppen, war aber nicht gelungen und die Kampffähigkeit der österreichisch-ungarischen Truppen nahm in den folgenden Monaten zunehmend $\mathrm{ab}^{(17)}$. Der Versuch einer Wiederholung des Erfolges im Juni 1918 scheiterte. Am ersten Jahrestag des Beginns der Offensive von Flitsch-Tolmein begann die Entente ihre letzte Offensive, womit, wie Rauchensteiner betont, „der 24. Oktober für Italien wie für das alte Österreich zu einem unvergesslichen Jahrestag“(18) wurde. Der Erfolg der Schlacht konnte den militärischen Zusammenbruch also bestenfalls ein wenig hinauszögern.

\section{Das Jahr 1917 in Überblickswerken zur österreichischen Geschichte}

1917 ist also in vielfältiger Hinsicht als „Schlüsseljahr“ für die Entwicklung Österreich(-Ungarn)s zu klassifizieren. Dies bestätigt ein Blick in das umfangreichen Überblickswerk Der Erste Weltkrieg und das Ende der Habsburgermonarchie ${ }^{(19)}$ (1222 Seiten) von Rauchensteiner, in dem - wenig überraschend aufgrund des zur Verfügung stehenden Platzes - alle drei oben genannten Entwicklungen ausführlich besprochen werden. Dass jedoch auch mit deutlich weniger Platz all diese Ereignisse erklärt werden können, zeigt die Kurzfassung des Buches Der Erste Weltkrieg und das Ende der Habsburgermonarchie in aller Kürze (276 Seiten) von Manfried Rauchensteiner und Josef Broukal ${ }^{(20)}$.

Daran, dass alle drei Entwicklungen gut erforscht sind, besteht also kein Zweifel, doch sind sie auch Teil des Funktionsgedächtnisses, das im Gegensatz zum Speichergedächtnis „eine Auswahl herstellt, die für lebendige Gedächtnisse erinnerbar ist, ein Identitätsangebot macht und Orientierungsfunktion besitzt“? ${ }^{(21)}$ Zur Konstruktion eines solchen dienen weniger solche wissenschaftliche Spezialstudien. In Gesamtdarstellungen bzw. Überblickswerken zur Geschichte Österreichs sind Wissenschaftler viel mehr gezwungen, aus der Fülle an Forschungsergebnissen jene auszuwählen, die sie für besonders relevant halten. Dies ergibt sich aus gewissen Wissenschaftstraditionen, aus gesellschaftlichen Diskursen und dem „Zeitgeist“. Sie dienen oft als

17 Franz Felberbauer, „Die 12. Isonzoschlacht: Der Operationsplan und seine Durchführung“, in: Manfried Rauchensteiner (Hg.), Waffentreue. Die 12. Isonzoschlacht 1917. Begleitband zur Ausstellung des Österreichischen Staatsarchivs, 23. Oktober 2007-1. Februar 2008, Wien, 2007, S. 13-33.

18 Manfried Rauchensteiner, „Einleitung“, in: Manfried Rauchensteiner (Hg.), Waffentreue. Die 12. Isonzoschlacht 1917. Begleitband zur Ausstellung des Österreichischen Staatsarchivs, 23. Oktober 2007-1. Februar 2008, Wien, 2007, S. 3-12, hier S. 11.

19 Manfried Rauchensteiner, Der Erste Weltkrieg und das Ende der Habsburgermonarchie, Wien/ Köln/Weimar, 2013.

20 Rauchensteiner/Broukal, Der Erste Weltkrieg (Anm. 9).

21 Aleida Assmann, „Speichern oder Erinnern? Das kulturelle Gedächtnis zwischen Archiv und Kanon“, in: Moritz Csáky, Peter Stachel (Hg.), Speicher des Gedächtnisses. Bibliotheken, Museen, Archive, Teil 2: Die Erfindung des Ursprungs. Die Systematisierung der Zeit, Wien, 2001, S. 15-29, hier S. 22. 
Prüfungslektüre, definieren also, was von Absolventen eines bestimmten Fachs an Wissen erwartet wird. Sie sind folglich geeignet für eine erste Annäherung an die Frage, inwiefern dem Jahr 1917 in Österreich besondere Bedeutung beigemessen wird und welche Ereignisse mit diesem insbesondere verbunden werden.

Erich Zöllners Geschichte Österreichs war für viele Studierendengenerationen die Lerngrundlage schlechthin. Im Kapitel „Der erste Weltkrieg“ werden die Ereignisse chronologisch besprochen - zum Jahr 1917 werden erwähnt die Proklamierung des uneingeschränkten U-Bootkrieges und die darauffolgende Kriegserklärung der USA, die russische „Märzrevolution“, die Sixtus-Affäre, der Sieg des Bolschewismus in Russland und die Entwicklung an den verschiedenen Fronten inkl. der Zwölften Isonzoschlacht, nicht jedoch die Wiedereinberufung des Reichsrates.

In Karl Vocelkas Geschichte Österreichs findet sich eine „Chronologie des Ersten Weltkrieges“, wo zum Jahr 1917 die Ereignisse „Uneingeschränkter U-Boot-Krieg Durchbruch am Isonzo, Rückzug der Italiener hinter die Piave - Kriegseintritt der USA - Oktoberrevolution in Russland“ genannt werden ${ }^{(22)}$. Im Text erklärt Vocelka in Bezug auf das Jahr 1917 nur die Folgen der Entwicklungen in Russland und den Kriegseintritt der USA ${ }^{(23)}$.

Brigitte Mazohl konzentriert sich in ihrem Artikel „Die Habsburgermonarchie 1848-1918“ in der von Thomas Winkelbauer herausgegebenen aktuellsten Geschichte Österreichs stärker auf die innere Entwicklung der Monarchie. In einer Zeittafel mit dem Titel „Der Weg in die Katastrophe und das Ende der Monarchie“ werden zum Jahr 1917 drei Ereignisse angeführt:

„16. April: Kriegseintritt der USA

,Sixtus-Affäre

30. Mai: Wiedereinberufung des Reichsrates“(24).

Im Text wird auch die Februarrevolution erwähnt, wobei die Entwicklungen aber wohl zu stark verkürzt wurden, wenn behauptet wird: „In Russland führten die Folgen des Krieges zur sogenannten Februarrevolution (nach Gregorianischem Kalender: 8. März 1917), die die Zarenherrschaft stürzte. In der Folge kam es zu Friedensverhandlungen zwischen Deutschland und Russland im Dezember 1917 (abgeschlossen am 3. März 1918) bei Brest-Litowsk, denen sich Österreich-Ungarn anschloss“(25). Außerdem wird im Text auf die Zwölfte Isonzoschlacht eingegangen; die Wiedereinberufung des Reichsrates wird hingegen nicht erwähnt.

In Überblickswerken, die sich mit der Geschichte Österreichs beschäftigen, wird also nicht immer auf alle die österreichische Entwicklung speziell betreffenden Ereignisse eingegangen; die meiste Aufmerksamkeit wird jenen beiden Vorgängen geschenkt, die weltpolitische Bedeutung hatten. Diese Schwerpunktsetzung lässt vermuten, dass auch in Darstellungen, die sich an eine breite Bevölkerungsschicht

22 Karl Vocelka, Geschichte Österreichs. Kultur-Gesellschaft-Politik, Graz/Wien/Köln, 2000 (2. Aufl.), S. 268.

23 Ibid., S. 270.

24 Brigitte Mazohl, „Die Habsburgermonarchie 1848-1918“, in: Thomas Winkelbauer (Hg.), Geschichte Österreichs, Stuttgart, 2015, S. 392-475, hier S. 440.

25 Ibid., p. 446. 
richten, vornehmlich auf die Entwicklungen in Russland sowie den Kriegseintritt der USA eingegangen wird.

\section{Das Jahr 1917 im Museum}

Macht man sich auf die Suche nach dem Jahr 1917 in österreichischen Ausstellungen, dann stellt man, wie bei vielen anderen für die Weltgeschichte bedeutenden Jahreszahlen und Ereignissen, schnell fest, dass es in Österreich (noch) kein nationales Geschichtsmuseum gibt ${ }^{(26)}$. Die umfangreichste Dauerausstellung zur Geschichte des eigenen Landes befindet sich derzeit im Heeresgeschichtlichen Museum in Wien. Dort gibt es seit 2014 eine neue Ausstellung, die sich auf rund 1400 Quadratmetern eingehend mit dem Ersten Weltkrieg beschäftigt ${ }^{(27)}$. Die Ausstellung ist chronologisch gegliedert, doch sind die verschiedenen Themenabschnitte, wie etwa „Gebirgskrieg“, oft nicht nur einem Kriegsjahr zuzuordnen. Um die wichtigsten Ereignisse pro Kriegsjahr zusammenzufassen, gibt es zu allen eine Chronologie. Diese konzentrieren sich stark auf jene Ereignisse, die den Krieg beeinflussen - folglich werden in jener zum Jahr 1917 unter anderem alle drei Isonzoschlachten dieses Jahres, die Entwicklungen in Russland und die Kriegserklärungen der USA an das Deutsche Reich und an Österreich-Ungarn angeführt; auch die Friedensinitiative von Kaiser Karl wird erwähnt, nicht jedoch die Wiedereröffnung des Reichsrates. Der Text, der neben einer Karte zur militärischen Lage in Mitteleuropa platziert ist, gibt einen Überblick über die Entwicklung an allen Fronten, wobei der Kriegseintritt der USA als „[1]etztlich kriegsentscheidend“ bewertet wird. Zum Ende des Krieges an der Ostfront findet sich schließlich noch ein eigener Ausstellungsbereich „Waffenstillstand“, ähnlich wie er auch zu anderen Ereignissen, wie etwa zur „Brussilow-Offensive und [zum] Kriegseintritt Rumäniens“ zu finden ist. Damit gibt es in der Ausstellung keinen besonderen Fokus auf das Jahr 1917, keine Hervorhebung als „Wendejahr“, jedoch werden alle für die militärische Entwicklung aus der Sicht Österreich-Ungarns einschneidenden Ereignisse genannt.

In den Ausstellungen im Schloss Schönbrunn und in der Hofburg in Wien, den Wohnsitzen des Kaiserhauses, finden sich hingegen nur sehr wenige Hinweise auf den Ersten Weltkrieg, auf die Ereignisse des Jahres 1917 wird nicht eingegangen. Anders ist das auf der von der Schloß Schönbrunn Kultur- und Betriebsges.m.b.H. betriebenen virtuellen Ausstellung Der Erste Weltkrieg und das Ende der Habsburgermonarchie, eine Unterseite von Die Welt der Habsburger. Wie der Screenshot zeigt, kann man sich mit Hilfe einer Zeitleiste näher mit allen Kriegsjahren befassen. Auch hier erfolgt keine Hervorhebung des Jahres 1917, doch finden sich auf dieser Übersicht Quellen, die auf die Entwicklung in Russland, auf den Kriegseintritt der USA und die Wiedereinberufung des Reichsrates hindeuten - zu all diesen gibt es auch Überblickstexte.

26 Zu den Plänen und Debatten um ein „Haus der Geschichte“ vgl. u.a. Andrea BraIt, „Fragen zum Verbleib von Expertisen. Zur Engführung der Diskussionen um ein Haus der Geschichte Österreich“, in: Thomas Winkelbauer (Hg.), Haus? Geschichte? Österreich? Ergebnisse einer Enquete über das neue historische Museum in Wien, Wien, 2016, S. 269-297 und die dort zitierte Literatur.

27 U.a. Christoph Hatscheк, „Im Angesicht des Todes ...“, neues Museum, 4 (2014), S. 50-57; Andrea Brait, „Cultures de la mémoire de la Première Guerre mondiale en Autriche. Le ,Jubilé 1914/2014“, Matériaux pour l'histoire de notre temps, 113 (2014), S. 6-14. 
Sucht man auf der Website gezielt, dann findet man ebenso Artikel zu den SixtusBriefen $^{(28)}$ und zur 12. Isonzoschlacht ${ }^{(29)}$.

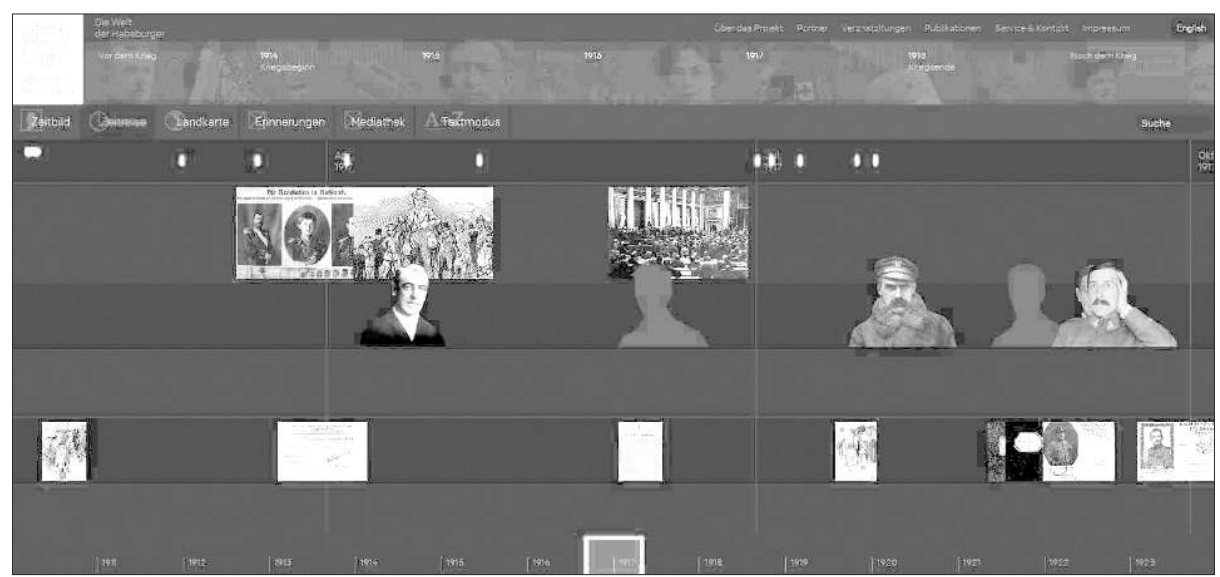

http://ww1.habsburger.net/de/zeitreise?jahr=1917

(c) Schloß Schönbrunn Kultur- und Betriebsges.m.b.H.

\section{Das Jahr 1917 in österreichischen Schulbüchern}

Der österreichische Schulbuchmarkt ist sehr vielfältig, was sich teilweise aus den unterschiedlichen Lehrplänen für die verschiedenen Schultypen ergibt. Die für die bestimmten Schultypen (immer für das gesamte Bundesgebiet) zugelassenen Schulbücher ${ }^{(30)}$ werden auf Schulbuchlisten erfasst und können von den Lehrkräften über die „Schulbuchaktion“ bestellt werden, die allen Schülern einen kostenlosen Zugang zu den Büchern ermöglicht ${ }^{(31)}$. Die großen Schulbuchverlage bieten zum Teil mehrere Lehrwerke pro Gegenstand und Schulstufe an; manche Lehrwerke sind mit geringfügigen Änderungen für verschiedene Schultypen zugelassen. Da pro Schüler nur ein limitiertes Budget (abhängig von Schultyp und Schulstufe) zur Verfügung steht, müssen die Verlage bei der Produktion darauf achten, dass die Bücher für die Schulen auch leistbar sind. Damit werden insbesondere die Seitenzahlen begrenzt, was sich wiederum auf die in den Schulbüchern vorzufindenden Inhalte auswirkt. Die Reduktion in der Darstellung ist also nicht nur eine didaktische Notwendigkeit, sondern auch eine finanzielle.

Aufgrund der großen Vielfalt konzentriert sich die folgende Analyse auf jene Bücher, die (im Schuljahr 2015/16) für die Allgemeinbildenden Höheren Schulen (AHS) zugelassen waren. Viele Lehrwerke für die Sekundarstufe I kommen auch in

28 http://wwl.habsburger.net/de/kapitel/die-sixtus-briefe-karls-suche-nach-einer-exitstrategie (online am 12. Mai 2016).

29 http://wwl.habsburger.net/de/kapitel/pyrrhus-sieg-und-scheitern-am-isonzo (online am 12. Mai 2016).

30 https://www.schulbuchaktion.at/schulbuchlisten.html (online am 17. September 2015).

31 Rupert SchredL, „Die Entwicklung der österreichischen Schulbuchaktion“, Erziehung und Unterricht, 4/145 (1995), S. 248-253, hier S. 248. 
Neuen Mittelschulen (NMS) und viele der Bücher für die Sekundarstufe II ebenso in Berufsbildenden Höheren Schulen (BHS) zum Einsatz. Jene Werke, die nur für NMS oder (einzelne) BHS zugelassen sind, wurden nicht berücksichtigt ${ }^{(32)}$.

Die Untersuchung folgt einer klassischen Form der Schulbuchanalyse, bei der die Wissenschaftsadäquatheit im Zentrum steht. Wiewohl, wie Bodo von Borries zu Recht betont, Schüler nicht zu kleinen Geschichtswissenschaftlern herausgebildet werden sollen ${ }^{(33)}$ und sich die Schulbuchforschung mittlerweile viel stärker auf die Formen der didaktischen Umsetzung konzentriert, ist doch festzuhalten, dass die in Schulbüchern festgehaltenen Inhalte und Interpretationen oft die einzigen und fast immer die ersten Geschichtsdarstellungen sind, mit denen die Lernenden konfrontiert werden. Auch in Zeiten der Kompetenzorientierung sind die in Schulbüchern tradierten Geschichtsbilder nicht beliebig geworden - das Arbeitswissen hat nach wie vor seinen Stellenwert im Unterricht, ist grundlegend für jede Kompetenzförderung. Diesem Grundgedanken folgend wird nachfolgend eine Analyse der „empirischen Triftigkeit“, also der „fachliche[n] Stimmigkeit der in historischen Narrationen getroffenen Aussagen“ vorgenommen. Empirische Triftigkeit wird, wie Waltraud Schreiber betont, beispielsweise „durch Abgleichen mit dem Forschungsstand sichergestellt, zeigt sich nicht zuletzt an der gewählten Fragestellung, an der schlüssigen Verwendung von Fachterminologie, an der methodischen Regulierung des Umgangs mit Vergangenheit/Geschichte mit Hilfe wissenschaftlicher oder zumindest wissenschaftsnaher Verfahrensweisen“(34).

Vergleicht man die verschiedenen AHS-Lehrwerke ${ }^{(35)}$ systematisch, dann fällt sofort auf, dass in allen die beiden für die internationale Entwicklung bedeutenden Ereignisse (der Kriegseintritt der USA und die Entwicklungen in Russland) zumindest kurz erwähnt werden. Von den hauptsächlich die Habsburgermonarchie betreffenden Ereignissen werden ab und zu die Friedensbemühungen von Kaiser Karl erklärt und ebenso selten der Durchbruch am Isonzo. Auf die Wiedereröffnung des Reichsrates wird in keinem einzigen Lehrwerk eingegangen.

Diese Aufstellung sagt freilich nur etwas über die Gewichtung der verschiedenen Themen aus ${ }^{(36)}$. Betrachtet man die aktuellen Lehrwerke für die Sekundarstufe I genauer, dann zeigt sich, dass ein Teil das Jahr 1917 als „Wendejahr“ interpretiert, das teilweise auch so bezeichnet wird. Im Schulbuch Zeitenblicke 3 findet sich etwa ein

32 Der Zugang zu den Lehrwerken erfolgte über Universitätsbibliotheken und die Schulbuch- und Schulschriftensammlung. Es wurde dabei versucht, die jeweils letzte Auflage zu berücksichtigen, was jedoch nicht in allen Fällen geprüft werden konnte.

33 Bodo von Borries, „Empirie der Schulbuchbewertung und Schulbuchbenutzung“, in: Christoph Kühberger, Philipp Miтtniк, Empirische Geschichtsschulbuchforschung in Österreich, Innsbruck/ Wien/Bozen, 2015, S. 19-41, hier S. 19.

34 Waltraud SchreIBER, „Geschichtstheoretische und Geschichtsdidaktische Grundlagen“, in: Waltraud Schreiber, Alexander Schöner, Florian Sochatzy (Hg.), Analyse von Schulbüchern als Grundlage empirischer Geschichtsdidaktik, Stuttgart, 2013, S. 9-37, hier S. 15.

35 Berücksichtigt wurden aufgrund der Vorgaben der aktuellen Lehrpläne die Bände für die 7. und 8. Schulstufe (3. und 4. Klasse AHS) und die 10. und 11. Schulstufe (6. und 7. Klasse AHS).

36 Eine genauere Analyse zum Umfang der einzelnen Themen könnte noch genauere Aufschlüsse bringen. 
Tabelle 1: Themen zu 1917 in österreichischen Schulbüchern (AHS)

\begin{tabular}{|c|c|c|c|c|c|}
\hline & $\begin{array}{c}\text { Kriegs- } \\
\text { eintritt USA }\end{array}$ & $\begin{array}{l}\text { Entwicklungen } \\
\text { in Russland }\end{array}$ & $\begin{array}{l}\text { Sixtus- } \\
\text { Briefe }\end{array}$ & $\begin{array}{c}\text { Wieder- } \\
\text { einberufung } \\
\text { des Reichsrates }\end{array}$ & $\begin{array}{c}12 . \\
\text { Isonzo- } \\
\text { schlacht }\end{array}$ \\
\hline \multicolumn{6}{|c|}{ Sekundarstufe I } \\
\hline Bausteine & $x$ & $x$ & $x$ & & \\
\hline $\begin{array}{r}\text { Durch die Vergangenheit } \\
\text { zur Gegenwart }\end{array}$ & $x$ & $x$ & & & $x$ \\
\hline Durch die Zeiten & $x$ & $x$ & & & \\
\hline einst und heute & $x$ & $x$ & & & \\
\hline ganz klar: Geschichte & $x$ & $x$ & & & \\
\hline Genial! Geschichte & $x$ & $x$ & & & \\
\hline Geschichte für alle & $x$ & $x$ & & & \\
\hline Geschichte live & $x$ & $x$ & & & $x$ \\
\hline Geschichte schreiben & $x$ & $x$ & $x$ & & \\
\hline Geschichte und Geschehen & $x$ & $x$ & & & \\
\hline Mehrfach Geschichte & $x$ & $x$ & & & \\
\hline $\begin{array}{r}\text { netzwerk geschichte @ } \\
\text { politik }\end{array}$ & $x$ & $x$ & & & \\
\hline ZeitenBlicke & $x$ & $x$ & & & \\
\hline Zeitbilder & $x$ & $x$ & & & \\
\hline Zeitfenster duo & $x$ & $x$ & & & \\
\hline \multicolumn{6}{|c|}{ Sekundarstufe II } \\
\hline geschichte.aktuell & $x$ & $x$ & $x$ & & \\
\hline gestern heute morgen & $x$ & $x$ & & & $x$ \\
\hline Go! Geschichte Oberstufe & $x$ & $x$ & & & \\
\hline netzwerk geschichte & $x$ & $x$ & $x$ & & $x$ \\
\hline Zeitbilder & $x$ & $x$ & & & \\
\hline Zeitfenster & $x$ & $x$ & $x$ & & $x$ \\
\hline
\end{tabular}

zweiseitiges Kapitel „1917 - ein Wendejahr der Weltgeschichte“(37), in Geschichte für alle 3 gibt es im Kapitel „Ausbruch und Verlauf des Ersten Weltkriegs“ das Unterkapitel „1917 - das Jahr der Wende“(38). Das Buch Geschichte und Geschehen 3 titelt „Die Wende im Krieg - das Epochenjahr 1917“(39) und auch in MEHRfach Geschichte 3. Teil 1: Wissen \& Verstehen wird der Begriff „Wendejahr“ verwendet. Hier heißt es im

37 Karl Vocelka, Andrea Scheichl, Christian Matzka, ZeitenBlicke 3. Geschichte und Sozialkunde. Politische Bildung, Wien, 2015 (2. Aufl.), S. 158f.

38 Elisabeth Monyk, Eva Schreiner, Elisabeth Mann, Geschichte für alle. 3. Klasse, Wien, 2015 (6. Aufl.), S. 149 (Hervorhebung im Original).

39 Gerhard Donhauser, Ludwig Bernlochner, Geschichte und Geschehen 3, Wien, 2008, S. 166. 
Kapitel „Der Erste Weltkrieg (1914-1918)“: „Wendejahr 1917. Die russische Revolution führte dazu, dass Russland die Entente verließ. Zugleich traten die USA in den Krieg ein “(40). In den Oberstufenbüchern GO! Geschichte Oberstufe $6^{(41)}$ und Zeitbilder $5 \& 6^{(42)}$ wird die Bewertung des Jahres 1917 den Lernenden überlassen.

Der Kriegseintritt der USA sowie die Umbrüche in Russland werden also in den meisten Schulbüchern als entscheidend für den Verlauf des Ersten Weltkrieges gesehen. Jedoch werden die beiden Ereignisse unterschiedlich (genau) erklärt. Oft gibt es zu den Ereignissen detailreiche Informationen, wie im Band Durch die Zeiten 3, wo erklärt wird: „Die russischen Niederlagen und die schwierige Versorgungslage der Bevölkerung führten 1917 zum Sturz des Zaren. Die neue russische Regierung setze den Krieg fort. Erst nachdem die bürgerliche Regierung durch eine kommunistische Revolution unter Führung Lenins gestürzt worden war, beendete Russland den Krieg und akzeptierte die harten Friedensbedingungen Deutschlands“(43). Im Folgeband Durch die Zeiten 4 werden im Kapitel „Die Sowjetunion - eine kommunistische Diktatur“ die Entwicklungen des Jahres 1917 dann noch einmal ausgeführt ${ }^{(44)}$. Im Buch Zeitbilder 3 findet sich sogar ein zweiseitiges Kapitel „Revolution in Russland“(45) und im Folgeband Zeitbilder 4 wird auf „Stalins Gewaltherrschaft“ eingegangen ${ }^{(46)}$. Auffällig ist, dass die Ermordung der Zarenfamilie in nur sehr wenigen Texten erwähnt wird - eine Ausnahme stellt das Schulbuch Geschichte für alle 3 dar ${ }^{(47)}$.

Auch zum Kriegseintritt der USA finden sich unterschiedlich genaue Erklärungen. Während in manchen Schulbüchern genau auf die Ursachen eingegangen wird, wird im Lehrwerk ganz klar: Geschichte 3 nur erklärt, dass die Mittelmächte einen Mehrfrontenkrieg führen mussten, „ab 1917 auch gegen die USA“(48). Im Gegensatz dazu schießt die Erklärung im Buch gestern heute morgen 6 übers Ziel hinaus. Dort wird behauptet: „Dadurch weiteten sich die Kämpfe zu einem Weltkrieg aus [...]“(49). Dass zahlreiche außereuropäische Staaten bereits 1914 am Krieg beteiligt waren, unter

40 Margot Graf, Franz Halbartschlager, Martina Vogel-Waldhütter, MEHRfach Geschichte 3. Teil 1: Wissen \& Verstehen, Wien, 2015 (2. Aufl.), S. 64 (Hervorhebung im Original).

41 Anschließend an eine Chronik zum Kriegsverlauf findet sich der Arbeitsauftrag: „Begründen Sie, warum das Kriegsjahr 1917 als Entscheidungsjahr bezeichnet wird." Franz Melichar, Irmgard Plattner, Claudia Rauchegger-Fischer, GO! Geschichte Oberstufe 6, Wien, 2012, S. 172.

42 Ein Arbeitsauftrag lautet: „Erarbeite Gründe, warum dem Jahr 1917 im Ersten Weltkrieg eine besondere Bedeutung zukommt.“ Alois Scheucher, Josef Scheipl, Eduard Staudinger, Ulrike Ebenносн, Zeitbilder 5 \&6. Geschichte und Sozialkunde. Politische Bildung. Von den frühen Hochkulturen zum Ende des Ersten Weltkrieges, Wien, 2011, S. 231.

43 Bernd Vogel, Birgit Wallner, Durch die Zeiten 3, Wien, 2006, S. 92 (Hervorhebung im Original).

44 Bernd Vogel, Birgit Wallner-Strasser, Durch die Zeiten 4, Wien, 2007, S. 28.

45 Alois Scheucher, Anton Wald, Ulrike Ebenhoch, Zeitbilder 3. Vom Beginn der Neuzeit bis zum Ende des Ersten Weltkrieges. Geschichte, Sozialkunde und Politische Bildung, Wien, 2013, S. 74f.

46 Ulrike Ebenhoch, Alois Scheucher, Anton Wald, Zeitbilder 4. Vom Ende des Ersten Weltkrieges bis zur Gegenwart, Wien, 2014, S. $20 \mathrm{f}$.

47 Elisabeth Monyк, Eva Schreiner, Elisabeth Mann, Geschichte für alle. 3. Klasse, Wien, 2015 (6. Aufl.), S. 149.

48 Ines PAPER, ganz klar: Geschichte 3, Wien, 2007, S. 90.

49 Oskar Achs, Manfred Scheuch, Eva Tesar, gestern heute morgen 6. Vom Beginn der Neuzeit bis zum Ende des Ersten Weltkriegs, Wien, 2004, S. 165. 
anderem Kanada und Australien (beide auf der Seite der Entente), muss in einem Schulbuch nicht unbedingt erwähnt werden; gleichzeitig sollte jedoch der falsche Eindruck vermieden werden, dass erst durch den Kriegseintritt der USA von einem Weltkrieg zu sprechen ist.

Dass 1917 an der Isonzofront ein militärischer Erfolg erreicht werden konnte, wird zwar manchmal erwähnt, jedoch wird meist ausgeblendet, dass dieser nur mit Hilfe deutscher Truppen und unter Einsatz von Giftgas erreicht werden konnte. So findet sich etwa auf einer Zeitleiste im Buch Durch die Vergangenheit zur Gegenwart 3 der Eintrag „Durchbruch der Österreicher an der Südfront“(50). Eine ähnliche Interpretation bietet Geschichte live 3, wo im Kapitel „Der Kriegsverlauf“ im Abschnitt zur Südfront erklärt wird, dass es „den Österreichern knapp vor Kriegsende [gelang], die italienische Front zu durchbrechen“(51).

Auf die Friedensbemühungen von Kaiser Karl wird selten genauer eingegangen. Im Schulbuch Bausteine 3 wird etwa nur kurz darauf verwiesen, dass Kaiser Karl sich erfolglos um Frieden bemühte, doch fehlen weitere Angaben ${ }^{(52)}$. Eine Ausnahme stellt der Oberstufenband geschichte.aktuell 1 dar ${ }^{(53)}$. In netzwerk geschichte 6 wird ebenfalls auf die Friedensbemühungen eingegangen, die jedoch als „dilettantisch aufgezogene Friedenssuche“ bewertet werden, als „ein Zeugnis diplomatischer Unfähigkeit sowohl für Kaiser Karl als auch für Außenminister Czernin“(54). Die ausführlichste Erklärung zu diesem Thema findet sich in Zeitfenster 6, jedoch kann diese nicht in allen Details einer wissenschaftlichen Prüfung standhalten, wenn etwa behauptet wird, dass die Verhandlungen an der italienischen Forderung, Südtirol abzutreten, gescheitert seien ${ }^{(55)}$. Im Band Geschichte und Geschehen 3 ist auffällig, dass als Ereignis in einer kurzen Übersicht genannt wird: „19. Juli 1917: Im Reichstag wird eine Friedensresolution geschlossen“. Diese wird dann aber nicht erklärt und es fehlt ebenso jeder Hinweis auf die Sixtus-Affäre ${ }^{(56)}$.

Der Zwang zur didaktischen Reduktion führt in vielen Schulbüchern zu einer verzerrten Darstellung, vielfach stimmen die Behauptungen nicht mit den historischen Abläufen überein, wobei sich manche Fehler leicht ausbessern ließen. So wird im Band Zeitfenster 4 duo im Kapitel „Die Sowjetunion unter Stalin“ behauptet: „Bereits 1917 war Russland unter der Führung Lenins mit dem Friedensvertrag von BrestLitowsk aus dem Ersten Weltkrieg ausgeschieden “(57), wobei an dieser Stelle nicht geklärt werden kann, ob es sich um einen Tippfehler bei der Jahreszahl handelt oder

50 Michael Lemberger, Durch die Vergangenheit zur Gegenwart 3, Linz, 2013 (4. Aufl.), S. 135.

51 Helmut Hammerschmid, Elfriede Windischbauer, Wolfgang Pramper, Geschichte live 3, Linz, 2009, S. 141.

52 Michael Bachlechner, Conny Benedik, Franz Graf, Franz Niedertscheider, Michael Senfter, Bausteine 3. Geschichte. Sozialkunde. Politische Bildung, Wien, 2012, S. 116.

53 Christian Roнr, geschichte.aktuell 1 für die AHS-Oberstufe, 5./6. Klasse, Linz, 2012 (4. Aufl.), S. 165.

54 Hans Pokorny, Renate Pokorny, Michael Lemberger, netzwerk geschichte 6, Wien, 2011 (2. Aufl.), S. 130.

55 Johannes Brzobohaty, Robert Salmeyer, Christa Zellhofer, Zeitfenster 6, Wien, 2011, S. 146-150.

56 Gerhard Donhauser, Ludwig Bernlochner, Geschichte und Geschehen 3, Wien, 2008, S. 166.

57 Ute Leonhardt, Robert Donner, Zeitfenster 4 duo. Geschichte. Sozialkunde. Politische Bildung, Wien, 2016, S. 22. 
der Friedensvertrag mit dem Waffenstillstand verwechselt wurde ${ }^{(58)}$. Dass aufgrund von zu starken Kürzungen die Abfolge von Ereignissen durcheinanderkommen kann, zeigt etwa eine Erklärung in einst und heute 3: „Er [Lenin] sollte in einer Revolution das Zarenreich stürzen und Frieden mit den Mittelmächten schließen “(59).

Umgekehrt zeigt sich aber auch, dass auf wenig Platz einfache und korrekte Erklärungen formuliert werden können. Ein „Auslassen“ mancher Details im Sinne der Verständlichkeit widerspricht der empirischen Triftigkeit nicht zwangsläufig. Dies zeigt etwa die ganz einfache, aber korrekte Aussage in Bausteine 3: „Nach der Revolution 1917 schloss Russland mit den Mächten einen Friedensvertrag“(60).

Wohl schwierig zu durchbrechen ist die Weitertradierung von bestimmten Interpretationen: So wird in allen Schulbüchern der Begriff „Oktoberrevolution“ verwendet, obwohl die Forschung längst davon ausgeht, dass es sich vielmehr um einen Staatsstreich gehandelt habe. Orlando Figes betont, dass „kaum ein historisches Ereignis [...] gründlicher durch Mythen verfälscht worden [sei] als das des 25. Oktober 1917“. Das vielfach tradierte Bild vom Kampf Zehntausender gehe hauptsächlich auf dem Film Oktober zurück, einen Propagandafilm aus dem Jahr 1928. Der „Sturm“ des Winterpalais, in dem das Karenski-Kabinett tagte, „ähnelte mehr einer gewöhnlichen häuslichen Festnahme “(61). Dies lässt sich in den Lehrwerken jedoch oft nur aus dem Kontext erschließen, eine Problematisierung des Begriffs „Revolution“ findet sich in keinem Buch. In manchen Werken ergibt sich ein deutlicher Widerspruch zwischen der Benennung der Ereignisse und den Erklärungen zu diesen, wie etwa im Lehrwerk GO! Geschichte Oberstufe 7, wo unter der Zwischenüberschrift „Die Oktoberrevolution“ zu lesen ist: „Durch Versprechen von Frieden und Landreform gelang es Lenin, seine Gegner im Petersburger Sowjet auszuschalten. Unterstützt von Teilen des Militärs gelang es ihm und Leon Trotzki, am 7. November 1917 (20. Oktober nach dem russischen Kalender) durch einen Putsch die Regierung Karenski zu stürzen und die Macht in der russischen Hauptstadt zu übernehmen“(62). Auf die unterschiedlichen Definitionen der Ereignisse wird nicht eingegangen. Dies ist vor allem deshalb sehr schade, weil die im Lehrplan geforderte „Sachkompetenz" auf einen kritischen und exakten Umgang

58 Ganz sicher um einen Fehler bei der Datierung handelt es sich bei folgender Ausführung: „Als das Deutsche Reich am 6. April 1917 [sic] den uneingeschränkten U-Bootkrieg ankündigte, fühlten sich die USA provoziert. Präsident Woodrow Wilson beendete aufgrund des uneingeschränkten U-Bootkriegs die diplomatischen Beziehungen zum Deutschen Reich. Am 6. April 1917 erklärten die USA dem Deutschen Reich den Krieg." Jutta Hofer, Bettina PAIreder, netzwerk geschichte @politik 3, Linz, 2013 (2. Aufl.), S. 134.

59 Gerhard Huber, einst und heute. chronologisch 3. Geschichte und Sozialkunde/Politische Bildung, Wien, 2012, S. 162. Im Folgeband findet sich zu den Umbrüchen des Jahres 1917 keine Erklärung mehr. Gerhard Huber, Ernst Gusenbauer, einst und heute. chronologisch 4. Geschichte und Sozialkunde/Politische Bildung, Wien, 2012, S. 25.

60 Michael Bachlechner, Conny Benedik, Franz Graf, Franz Niedertscheider, Michael Senfter, Bausteine 3. Geschichte. Sozialkunde. Politische Bildung, Wien, 2012, S. 116.

61 Orlando Figes, Die Tragödie eines Volkes. Die Epoche der russischen Revolution 1891 bis 1924, Berlin, 1998, S. 512.

62 Franz Melichar, Irmgard Plattner, Claudia Rauchegger-Fischer, GO! Geschichte Oberstufe 7, Wien, 2013, S. 18 (Hervorhebung im Original). 
mit Fachbegriffen abzielt ${ }^{(63)}$. Sie nimmt, wie Christoph Kühberger betont, „Bezug auf Begriffe, die das historische Denken strukturieren und begleiten, und betrifft Konzepte, die hinter derartigen (Fach-)Begriffen stehen“(64). Eine Diskussion der verwendeten Begrifflichkeiten im Hinblick auf die mit Quellen nachweisbaren Prozesse (beispielsweise Fotos, die zeigen, wie klein die Gruppe der Aufständischen war ${ }^{(65)}$ ) sowie Geschichtsdarstellungen (etwa das oft in Schulbüchern abgedruckte Gemälde von Wladimir Serow „Lenin proklamiert die Sowjetmacht “(66) ) wären eine gute Gelegenheit zur Schärfung des Revolutionsbegriffs sowie zur Abgrenzung von den Konzepten des Staatsstreichs bzw. des Putsches. In manchen Büchern wird der Begriff der „Oktoberrevolution“ mit Anführungszeichen verwendet, um anzudeuten, dass dieser Begriff für die beschriebenen Ereignisse üblich sei, wie etwa im Band Geschichte schreiben 4, wo es heißt: „Im November 1917 stürzten die Bolschewiki die Regierung und übernahmen die Macht (,Oktoberrevolution'). Dies war der Beginn der kommunistischen Diktatur in Russland“(67). Dass man auch ohne den problematischen Begriff „Oktoberrevolution“ auskommen kann, zeigt das Schulbuch Durch die Zeiten 4. Hier heißt es im Unterkapitel „Revolution und Bürgerkrieg“ zum Kapitel „Die Sowjetunion - eine kommunistische Diktatur“: „Im Oktober 1917 besetzten die Roten Garden, die Parteisoldaten der Kommunisten, die wichtigsten Gebäude von Sankt Petersburg und vertrieben die bürgerliche Regierung. Unter Führung Lenins ignorierten die Kommunisten den Ausgang der abgehaltenen Wahlen und verdrängten mit Terrormethoden alle anderen Parteien aus den Sowjets“(68).

63 Vgl. dazu grundlegend: Alexander ScHönER, „Kompetenzbereich historische Sachkompetenzen“, in: Andreas Körber, Waltraud Schreiber, Alexander Schöner (éd.), Kompetenzen Historischen Denkens. Ein Strukturmodell als Beitrag zur Kompetenzorientierung in der Geschichtsdidaktik, Neuried, 2007, S. 265-314.

64 Vgl. hierzu genauer u.a.: Christoph KüHBERger, Kompetenzorientiertes historisches und politisches Lernen. Methodische und didaktische Annäherungen für Geschichte, Sozialkunde und Politische Bildung, Innsbruck/Wien/Bozen, 2015 (3. überarbeitete und erweiterte Auflage), S. 78.

65 O. Figes, Die Tragödie (Anm. 61), S. 522.

66 Vgl. zu diesem Gemälde: Michael Worbing, „Wladimir A. Serow: Lenin proklamiert die Sowjetmacht 1917“, in: Michael Wor Bing, Susanne Popp, Der europäische Bildersaal. Europa und seine Bilder. Analyse und Interpretation zahlreicher Bildquellen, Schwalbach/Ts., 2014, S. 110-121.

Vielfach fehlen in den Büchern nähere Informationen zu diesem Gemälde. Das Schulbuch Genial! Geschichte 4 datiert dieses ins Jahr 1917 und blendet damit völlig aus, dass es sich um ein nach dem Zweiten Weltkrieg entstandenes Propagandabild handelt. Christine Baumgartner-Lemberger, Genial! Geschichte 4. Geschichte und Sozialkunde/Politische Bildung. Lehr- und Arbeitsbuch für die 8. Schulstufe, Wien, 2015 (2. Aufl.), S. 39. Hingegen finden sich im Lehrwerk Durch die Vergangenheit zur Gegenwart 4 hilfreiche Arbeitsaufträge zur Dekonstruktion: „Was ist auf dem Gemälde von Wladimir Serow aus dem Jahr 1947 zu sehen? Aus welcher Sichtweise wurde das Bild geschaffen? Welche Stimmung wird hier vermittelt?" Michael Lemberger, Durch die Vergangenheit zur Gegenwart 4, Linz, 2009, S. 25. Dass diese noch nicht mit Operatoren formuliert sind, erklärt sich aus dem Erscheinungsdatum dieses Schulbuchs.

67 Anneliese GIDL, Geschichte schreiben. Geschichte und Sozialkunde/Politische Bildung. Arbeitsbuch 3, Wien, 2013 (2. Aufl.), S. 23.

68 Bernd Vogel, Birgit Wallner, Durch die Zeiten 4, Wien, 2007, S. 28 (Hervorhebung im Original). 
Der Erste Weltkrieg wird den Lehrplänen entsprechend durchgehend in den Bänden für die 3. und für die 6. Klasse behandelt ${ }^{(69)}$; da in den Lehrplänen für die 4. und für die 7. Klasse jedoch die Behandlung von Diktaturen zwischen dem Ersten und Zweiten Weltkrieg vorgesehen ist ${ }^{(70)}$, wird vielfach in den Büchern für diese Klasse abermals (genauer, wiederholend oder nur kurz rückblickend) auf die Umbrüche in Russland 1917 eingegangen, bevor die Gründung der Sowjetunion dargestellt wird ${ }^{(71)}$. Durch den, den Lehrplänen entsprechenden, stark chronologischen Aufbau der Bücher ${ }^{(72)}$, wird die von der Forschung betonte Zäsur und die Langzeitentwicklung in Bezug auf die Umbrüche in Russland 1917 kaum deutlich. Im Schulbuch Zeitbilder 7 \& 8 wird jedoch zu Beginn des Kapitels „Russland: Vom Zarismus zur Sowjetunion“ betont: „Die Oktoberrevolution von 1917 und die Machtergreifung der Kommunisten in Russland gehören zu den wichtigsten historischen Ereignissen in der Geschichte des 20. Jh. “(73). Da sich die Erklärungen in diesem Kapitel nur bis zum Jahr 1922 erstrecken, wird die Bedeutung dieses Systemwandels jedoch nicht deutlich - diese ergibt sich erst viel später im Buch in den Kapiteln „Entwicklung und Ende des ,Kalten Krieges“" und „Weltmächte seit 1945“.

Der neue, ab Herbst 2016 gültige Lehrplan für die AHS-Unterstufe ${ }^{(74)}$ unternimmt einen deutlichen Versuch, von der bisher dominierenden Chronologie wegzukommen. Hier wird etwa im Modul 7 (3. Klasse) klar gefordert: „Politische Umbrüche, die als Revolutionen bezeichnet werden, vergleichen und bewerten“. In Modul 4 (3. Klasse) soll außerdem „die Problematik von Krieg, insbesondere des Ersten Weltkriegs, im Zusammenhang mit sich wandelnden europäischen Mächteverhältnissen, neuen Staatsordnungen und Nationalitätenkonflikten“ erläutert werden. Mit diesen Vorgaben ist auf jeden Fall ein Hinterfragen des Revolutionsbegriffs für die Umbrüche in Russland 1917 möglich. Ohne einen Längsschnitt zur Entwicklung der in der Folge etablierten Diktatur und die Folgen für die weltpolitische Ordnung - Stichwort „Kalter Krieg“ (der in diesem Lehrplan nicht erwähnt ist) - kann jedoch die von

69 Für die 3. Klasse ist im Lehrplan als „Kernbereich“ definiert: „Staaten und Völker im Krieg - Kriegsursachen und Friedensbemühungen in der Neuzeit bis zum Ersten Weltkrieg“, https://www.bmbf. gv.at/schulen/unterricht/lp/ahs11_786.pdf?4dzgm2 (online am 11. Mai 2016). Für die 6. Klasse ist vorgesehen: „Ursachen und Folgen des Ersten Weltkrieges“, https://www.bmbf.gv.at/schulen/unterricht/ lp/lp_neu_ahs_05_11857.pdf?4dzgm2 (online am 11. Mai 2016).

70 Für die 4. Klasse ist im Lehrplan festgeschrieben: „Entstehung und Bedingungen diktatorischer Systeme, Methoden totalitärer Herrschaft: Faschismus, Nationalsozialismus, Kommunismus“; für die 7. Klasse ist definiert: „demokratische, autoritäre und totalitäre Staatensysteme und ihre Ideologien (Systemvergleiche; Kommunismus, Faschismus, Nationalsozialismus; Radikalisierung des politischen Lebens in Österreich 1918-1938)“.

71 Eine Ausnahme stellt der Band Geschichte und Geschehen 4 dar, in dem auf das Jahr 1917 nicht mehr Bezug genommen, sondern „Die Diktatur Stalins“ in den 1920er- und 1930er-Jahren erklärt wird. Gerhard Donhauser, Ludwig Bernlochner, Geschichte und Geschehen 4, Wien, 2009, S. $30 \mathrm{f}$.

72 Die Reihe Geschichte Oberstufe versucht davon am ehesten wegzukommen und präsentiert viele Längsschnitte, ist aber freilich auch an den chronologisch strukturierten Lehrplan gebunden.

73 Eduard Staudinger, Alois Scheucher, Ulrike Ebenhoch, Josef Scheipl, Zeitbilder 7 \& 8. Geschichte und Sozialkunde. Politische Bildung. Vom Ende des Ersten Weltkrieges bis in die Gegenwart, Wien, 2012, S. 12. 
Wissenschaftlern diagnostizierte Bedeutung des Jahres 1917 nicht vermittelt werden. Das Schreiben eines Schulbuches, das dem Lehrplan, aktuellen didaktischen Vorstellungen (Kompetenzorientierung, Unterrichtsprinzipien u.s.w.) entspricht und gleichzeitig einer Triftigkeitsanalyse standhält, gleicht also auch künftig einer Quadratur des Kreises.

\section{Fazit}

Die Analyse hat gezeigt, dass den Entwicklungen des Jahres 1917 in den meisten untersuchten populärwissenschaftlichen Darstellungen eine große Bedeutung zugeschrieben wird. Der Schwerpunkt der Darstellungen liegt klar auf den Entwicklungen in Russland und dem Kriegseintritt der USA; hinsichtlich der die Habsburgermonarchie betreffenden Ereignissen fließen hauptsächlich jene Überblickswerke und populärwissenschaftliche Darstellungen ein, die in einem unmittelbaren Zusammenhang mit der Entwicklung des Weltkrieges stehen. Mit der Fokussierung auf die internationalen Entwicklungen ist eine klare Bewertung verbunden, wobei von einer längeren Tradition ausgegangen werden kann. Dies wäre in einer weiteren Studie zu prüfen, die ältere Ausstellungen und Lehrwerke einbezieht.

Aufgrund des zur Verfügung stehenden Platzes ist keiner populärwissenschaftlichen Darstellung ein Vorwurf zu machen, dass eine Auswahl getroffen werden muss und demnach nicht alle Entwicklungen dargestellt werden können - es können immer nur Vergangenheitspartikel dargestellt werden. Die Analyse zeigte jedoch überaus unterschiedlich empirisch triftige Aussagen - das Ergebnis ist wohl nicht nur ein Auftrag an Schulbuchautoren und Verlage sondern auch an die Fachwissenschaft, ihre Expertisen stärker in Lehrwerke für den Schulunterricht einfließen zu lassen.

\section{Zusammenfassung}

In umfangreichen geschichtswissenschaftlichen Werken wird das Jahr 1917 klar als Wendepunkt in der Geschichte dargestellt. Für die Habsburgermonarchie werden neben den Umbrüchen im Russischen Reich und dem Kriegseintritt der USA drei weitere bedeutende Ereignisse genannt: die Friedensbemühungen von Kaiser Karl I., die erfolgreiche Offensive der Mittelmächte in der 12. Isonzoschlacht und die Wiederöffnung des Reichsrates. Die Untersuchung hat jedoch gezeigt, dass in Darstellungen, die sich eher an ein Laienpublikum richten, hauptsächlich auf die beiden international bedeutenden Ereignisse eingegangen wird. In den analysierten österreichischen Schulbüchern fanden sich nicht nur stark verkürzte Darstellungen, sondern auch einige, die den historischen Abläufen widersprechen; außerdem werden zahlreiche Möglichkeiten zur Förderung fachspezifischer Kompetenzen nicht genützt.

\footnotetext{
Abstract

Comprehensive historical works clearly portray the year 1917 as a turning point in history. Regarding the Hapsburg monarchy they mention besides the radical change taking place in the Russian Empire, three further important events: the peace efforts of Emperor Charles I, the successful offensive of the Central Powers in the $12^{\text {th }}$ Isonzo battle, and the reopening of the Reichsrat. This study has shown, however, that in the case of historical
} 
publications which are more likely to address a lay public, the focus is mainly put on the two internationally significant events. Furthermore, the analysis of Austrian textbooks revealed that there could be found not only greatly shortened presentations of the events, but also some that clearly contradict the historical processes. Additionally one has failed to seize numerous support possibilities in order to promote specific skills.

\section{Résumé}

Dans bien des ouvres historiques exhaustives, l'année 1917 est clairement représentée comme un tournant dans l'histoire. En ce qui concerne la monarchie des Habsbourg, on cite, outre le changement radical ayant lieu dans l'Empire Russe, trois autres événements importants: les efforts de paix de l'empereur Charles I ${ }^{\mathrm{er}}$, l'offensive militaire réussie des Empires centraux dans la $12^{e}$ bataille d'Isonzo et la réouverture du Reichsrat. Cependant, l'étude a montré que dans le cas de publications qui sont plus susceptibles d'être dirigées vers un public lä̈que, le focus est davantage dirigé sur les deux événements internationaux les plus importants. En outre, on a dî constater que les manuels scolaires autrichiens analysés ne contiennent pas seulement des versions très raccourcies, mais également certaines qui contredisent clairement les processus historiques; de plus, de nombreuses possibilités de promotion pour développer des compétences spécifiques n'ont pas été saisies. 